\title{
Single-pixel terahertz imaging: a review
}

\section{Luca Zanotto ${ }^{1}$, Riccardo Piccoli ${ }^{1}$, Junliang Dong ${ }^{1}$, Roberto Morandotti ${ }^{1,2}$ and Luca Razzari ${ }^{1 *}$}

This paper is devoted to reviewing the results achieved so far in the application of the single-pixel imaging technique to terahertz $(\mathrm{THz})$ systems. The use of $\mathrm{THz}$ radiation for imaging purposes has been largely explored in the last twenty years, due to the unique capabilities of this kind of radiation in interrogating material properties. However, THz imaging systems are still limited by the long acquisition time required to reconstruct the object image and significant efforts have been recently directed to overcome this drawback. One of the most promising approaches in this sense is the so-called "single-pixel" imaging, which in general enables image reconstruction by patterning the beam probing the object and measuring the total transmission (or reflection) with a single-pixel detector (i.e., with no spatial resolution). The main advantages of such technique are that i) no bulky moving parts are required to raster-scan the object and ii) compressed sensing (CS) algorithms, which allow an appropriate reconstruction of the image with an incomplete set of measurements, can be successfully implemented. Overall, this can result in a reduction of the acquisition time. In this review, we cover the experimental solutions proposed to implement such imaging technique at $\mathrm{THz}$ frequencies, as well as some practical uses for typical THz applications.

Keywords: terahertz imaging; single-pixel imaging; compressed sensing

Zanotto L, Piccoli R, Dong J L, Morandotti R, Razzari L. Single-pixel terahertz imaging: a review. Opto-Electron Adv 3, 200012 (2020).

\section{Introduction}

The interest of the scientific community in using terahertz $(\mathrm{THz})$ radiation (frequency between $1-10 \mathrm{THz}$ ) for imaging purposes has been continuously growing in the last twenty years ${ }^{1}$. The reasons underlying such interest are the peculiar abilities of $\mathrm{THz}$ radiation to extract materials properties. For example, THz light can pass through a vast variety of optically opaque materials, such as semiconductors, plastics and papers ${ }^{2}$. This enables the recognition, with high contrast, of the presence of hidden items such as metals ${ }^{3}$, or the investigation of the inner structure of an object concealed by some external layers ${ }^{4}$. In addition, the significantly shorter wavelength in comparison to that of microwave radiation allows resolutions of the order of 100 microns at the diffraction limit $(\lambda=300 \mu \mathrm{m}$, at $1 \mathrm{THz})$, which can be further improved in near-field conditions ${ }^{5}$. Moreover, the $\mathrm{THz}$ photon energy is very low ( $\sim \mathrm{meV}$, non-ionizing), making the use of this radiation safe for both the human body and sensitive samples. Finally, $\mathrm{THz}$ spectroscopy can be exploited for chemical recognition of substances featuring rotational/vibrational transitions or collective excitations in this range of frequencies, including gases ${ }^{6}$, organic compounds, and biological molecules ${ }^{8}$. One of the most powerful techniques employed in $\mathrm{THz}$ technology is the so-called time-domain spectroscopy (TDS), which is based on the coherent detection (amplitude and phase of the electric field) of a broadband $\mathrm{THz}$ pulse. This measurement method allows the complete characterization of the $\mathrm{THz}$ properties of a material, giving direct access to the complex refractive index in such frequency range ${ }^{9}$. The application of THz-TDS to an imaging system readily enables the retrieval of the key optical parameters at each spatial position of the object under investigation. These unique

\footnotetext{
${ }^{1}$ Institut National de la Recherche Scientifique (INRS), Centre Énergie, Matériaux et Télécommunications (EMT), Varennes, QC J3X 1S2, Canada. ${ }^{2}$ Institute of Fundamental and Frontier Sciences, University of Electronic Science and Technology of China, Chengdu 610054, China.

*Correspondence: L. Razzari, E-mail: razzari@emt.inrs.ca

Received: 16 April 2020; Accepted: 22 June 2020; Published: 23 September 2020
} 
features opened the route to a great number of possible applications over the last decades, as it is demonstrated by the constant increase of the number of publications on the topic. Potential "real world" uses of such technology span from security monitoring ${ }^{3,10}$, to quality and safety control in industry $y^{4,11-13}$, biomedical applications ${ }^{14-16}$, and also art conservation ${ }^{17-19}$.

Nevertheless, a broader spread of $\mathrm{THz}$ imaging technologies is mostly hampered by the long time required for image acquisition. Indeed, most of the commonly used $\mathrm{THz}$ systems for imaging rely on detectors with no spatial resolution, which means that one is compelled to raster-scan the object pixel by pixel. For example, a relatively low resolution (for digital cameras) image of $640 \times 480$-pixel would require almost 1 hour when recorded with a state-of-the-art commercial THz-TDS system 20 . The need of mechanically displacing the sample or the overall $\mathrm{THz}$ generation-detection system to retrieve the image is another reason. Thus, many different solutions have been proposed to pursue the realization of $\mathrm{THz}$ imaging systems with a faster acquisition time ${ }^{21}$, which can be sorted into two main categories: i) systems exploiting innovative $\mathrm{THz}$ cameras and ii) techniques based on single-pixel detectors. Regarding the first, $\mathrm{THz}$ cameras based on thermal detector arrays, such as micro-bolometer ${ }^{22,23}$ or pyroelectric sensors ${ }^{24}$ have been developed. In addition, field-effect transistor cameras have been also realized ${ }^{25}$. Although these devices allow real-time operation, they lack temporal/spectral resolution, and suffer from low sensitivity. Considering instead single-pixel-detectorbased systems, the common approach, as mentioned above, is based on raster-scanning the object, which can be improved by using very fast mechanical beam steering devices ${ }^{26,27}$ and retrieving the $\mathrm{THz}$ waveform in time at each spatial position via, e.g., electro-optic sampling ${ }^{27}$. More advanced techniques implementing crosscorrelation (in the time or frequency domain) between the measured electric field and a reference can also be used to ameliorate the detection capabilities of typical THz-TDS systems ${ }^{28,29}$. One alternative method exploits the spectrum (or temporal waveform) of a $\mathrm{THz}$ pulse to encode the information of the object spatial structure ${ }^{30,31}$. Such an approach can effectively address the long acquisition time issue, but with loss of spectral information, being the frequency components employed to spatially interrogate the object. Finally, another possibility is the so-called single-pixel imaging scheme.
Single-pixel imaging systems have been widely studied at optical frequencies. Such technique relies on illuminating the object with a series of spatial light patterns and recording, for each one of them, the total transmitted or reflected light by means of a detector with no spatial resolution (i.e. with a single pixel). The correlation between the spatial distributions of the patterns and the corresponding measurements performed by the detector leads to the image retrieval ${ }^{32}$. Besides removing the need of mechanical motorized parts typically employed for raster-scanning, such technique allows the application of the compressed sensing (CS) theory, which generally defines the conditions under which it is possible to reconstruct an under sampled signal with good approximation ${ }^{33}$. When the signal is an image, this means performing a number of measurements smaller than the total number of retrieved pixels, which is made possible by the choice of an appropriate set of illuminating patterns, exploiting the sparsity of most typical objects ${ }^{33}$.

This paper is devoted to reviewing the research work performed in the last years on the application of the single-pixel scheme to $\mathrm{THz}$ imaging systems. The main experimental challenges are considered, as well as the performance of this scheme in typical $\mathrm{THz}$ imaging applications. Section 2 gives a general introduction on single-pixel imaging. Section 3 reports on different implementations of pattern generation at $\mathrm{THz}$ frequencies. Finally, Section 4 presents a series of experimental results, showing the application of the single-pixel imaging scheme in contexts where $\mathrm{THz}$ technology is typically employed.

\section{Single-pixel imaging and compressed sensing}

The concept of single-pixel imaging relies on using a series of multi-pixel light patterns to illuminate the object and then recording the total transmitted (or reflected) light by means of a single-pixel detector. Mathematically, this procedure corresponds to encoding the information of the object spatial distribution onto a vector representation, whose components are the projections of the object transmission/reflection function onto the basis vectors. The set of outcomes from the detector therefore corresponds to such components, in the chosen representation. One can rewrite the $n \times n$ object transmission/reflection function $T(x, y)$ in the form of a column $n^{2}$-element vector $S$, by placing the columns one 
after the other, and group the corresponding series of $n^{2}$ measurement from the detector into another vector $\boldsymbol{W}$. Each of the $n^{2}$ patterns $\left\{H_{i}(x, y)\right\}_{i=1, \ldots, n^{2}}$ can be also reshaped into a $n^{2}$-element vector by stacking the columns one after the other, and subsequently the set of vectors can be regrouped into a $n^{2} \times n^{2}$ matrix $\boldsymbol{H}$. In this fashion, the measurement process can be seen as a matrix-vector multiplication. The knowledge of the matrix $\boldsymbol{H}$ and the measurement vector $\boldsymbol{W}$ enables the retrieval of the vector $S$ (i.e., the image of the object), by simply solving a linear system of equations:

$$
W=\boldsymbol{H} \text {. }
$$

In general, solving this problem requires the dimension of vector $W$ to be equal to that of vector $S$, which in terms of measurements means that the number of patterns is equal to the number of pixels in the image. Considering the measurement matrix $\boldsymbol{H}$, a straightforward choice is represented by the identity matrix (Fig. 1(a)), whose corresponding patterns consist of only one illuminated pixel (Fig. 1(b)). Although in principle equivalent to the raster-scan technique, this choice is clearly very poor in terms of signal-to-noise ratio, since for every illumination pattern a very weak light intensity $\left(1 / n^{2}\right)$ is available. Evidently, a wide variety of pattern bases selections is possible, each of them providing a different performance under variable operating conditions. Two of the most studied bases are the Hadamard set (shown in Figs. 1(c)-1(d)) which guarantees an optimal signal-to-noise ratio $^{34}$, and the Fourier set, which shows a good efficiency when operating in CS conditions ${ }^{35,36}$.

As briefly mentioned above, one of the main advantages of the single-pixel imaging scheme is the related possibility of applying the so-called CS theory, which tackles the problem of solving an undetermined inverse problem in which the unknown values are more than the available data. Such a situation is usually encountered when one wants to estimate data values at temporal/spatial frequencies higher than the ones available from the sampling equipment ${ }^{37}$. In the specific case of single-pixel imaging, as mentioned earlier, this means retrieving an image with a number of patterns smaller than the total number of pixels. It is well-known that images are generally "compressible" under certain representations $s^{38}$. The compressibility of an image is related to the concept of "sparsity", which directly connects to the number of non-zero entries in the vector representing the image. Sparsity is already used, for instance, in JPEG compression to reduce the size of images in computers. In such a case, the compression is applied after the image is acquired via a digital camera by discarding all quasi-zero coefficients, corresponding to high-spatial frequency components in the discrete cosine representation (which express a signal in terms of a finite number of cosine functions with variable frequency $)^{33}$. In CS though, the compression is employed during the acquisition process in order to enable the reconstruction of the image with an "incomplete" set of measurements. In this situation, the problem presented in Eq. (1) is undetermined (i.e., it has infinite solutions), nevertheless it is possible to apply a pseudo-inversion in order to retrieve the image. Numerous strategies have been developed to pursue such reconstruction and the field is still an open subject of research ${ }^{32}$. The traditional approach, first proposed by Candès and $\mathrm{TaO}^{33,39}$, relies on using a sensing basis which is incoherent with the spatial features of the image (usually pseudo-random bases). Subsequently, an iterative optimization procedure enables the retrieval of the image. Typically, this kind of optimization algorithms are based on minimizing the $l^{1}$-norm, and it is demonstrated that the reconstruction is correct when the number of samples is $m \geq O(K \cdot \log (N / K))$, where $K$ is the sparsity and $N$ the
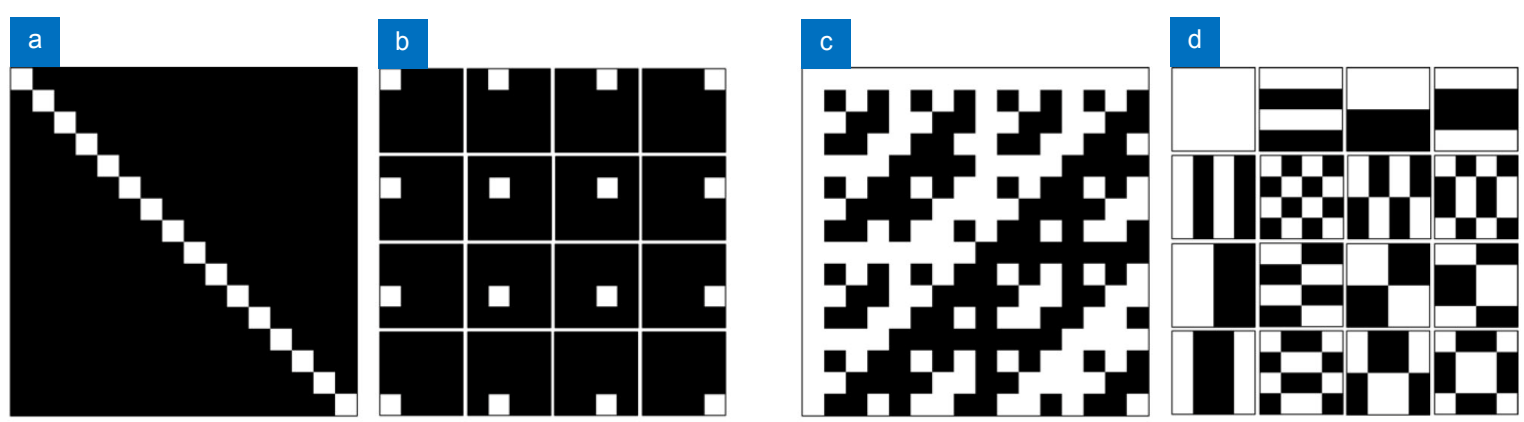

Fig. 1 | (a) $16 \times 16$ identity matrix $\boldsymbol{H}$ and (b) the corresponding series of $16\left(n^{2}\right), 4 \times 4$ spatial masks $\left\{H_{i}(x, y)\right\}_{i=1 \ldots 16}$ obtained from it; (c) $16 \times 16$ Hadamard matrix $\boldsymbol{H}$ and (d) corresponding set of $16,4 \times 4$ spatial masks $\left\{H_{i}(x, y)\right\}_{i=1 \ldots 16}$. 
number of pixels in the image ${ }^{40,41}$. Such algorithms usually provide a faithful reconstruction of the image, but they can be quite demanding in terms of computational burden. Alternative methods make use of sampling bases which are not incoherent with the spatial distribution of the image, like the Hadamard, Fourier or wavelet matrices. In these cases, a subset of the basis has to be chosen and the image is retrieved using a fast algorithm. This is generally preferable when the application needs fast reconstruction but does not require very high resolutions. Clearly, in such scenario it is important to choose a basis subset which properly samples the object spatial features. This can be done adaptively or with prior information about the object ${ }^{32}$.

In the context of this review, the main reason to apply a single-pixel imaging approach to the $\mathrm{THz}$ domain is to address the long acquisition time issue, while avoiding the need of multi-pixel detectors. Understanding the best operating conditions of CS for the specific case of $\mathrm{THz}$ imaging is therefore crucial. A recent study ${ }^{42}$, for example, has attempted to assess the performance of some single-pixel methods employing $\mathrm{THz}$ radiation. The authors considered different pattern bases, categorizing them as grey-scale or binary, and pseudo-random or deterministic. More specifically, pseudo-random Bernoulli and Gaussian masks, as well as deterministic Hadamard and discrete cosine transform (DCT) bases were compared. Their performance was mainly evaluated in terms of compressibility, by employing a continuous wave $(\mathrm{CW})$ source emitting at $0.35 \mathrm{THz}$.

The study concluded that, for the specific conditions considered, Bernoulli masks were the best choice. Notwithstanding this, the overall performance of a CS approach can greatly vary depending on the conditions of operation, for what concerns, for instance, the signal-to-noise ratio (SNR) of the system, the employed detection technique, the desired resolution and the required speed of the reconstruction. Thus, in a general sense, the selection of the pattern basis needs to be adapted to the specific use and targeted performance of the $\mathrm{THz}$ imaging system to be developed.

\section{Pattern generation for single-pixel $\mathrm{THz}$ imaging}

As said earlier, the central idea of single-pixel imaging is to use a series of multi-pixelated light patterns to illuminate the object under investigation. To this end, spatial modulation of the light beam is required. Spatial light modulators (SLMs) are common tools (based, for example, on micro-mirror devices or liquid crystals) that typically operate at visible/infrared frequencies. However, no commercial SLM is available that can directly modulate a beam in the $\mathrm{THz}$ frequency range. Thus, the choice of appropriate methods to pattern light at $\mathrm{THz}$ frequencies represents an important aspect for effectively implementing single-pixel $\mathrm{THz}$ imaging. Various solutions have been proposed in the last years: they are presented below, sorted by considering whether they are based on (i) the direct modulation of the $\mathrm{THz}$ beam or (ii) the patterned illumination of the nonlinear crystals used for the generation/detection of $\mathrm{THz}$ radiation.

\section{Direct modulation of the $\mathrm{THz}$ beam}

\section{Metallic masks}

The first solution proposed for $\mathrm{THz}$ spatial modulation made use of metallic binary masks shaped into the series of patterns needed to perform the reconstruction ${ }^{43}$. In this work, the masks were $32 \times 32$-pixel with $1 \times 1 \mathrm{~mm}^{2}$ pixel size, all with $\sim 50 \%$ of transmitting pixels, randomly chosen. These masks were placed on the $\mathrm{THz}$ beam path and acted as amplitude modulators (as shown in Fig. 2(a)). Physical masks offer an optimal modulation depth, being truly binary. The drawback is clearly the lack of flexibility and the need to mechanically slide them into the $\mathrm{THz}$ path, thus limiting the achievable patterning speed of the system. One improved implementation of this approach made use of a metallic spinning disk with a series of engraved patterns (see Fig. 2(b)), in order to increase the acquisition speed ${ }^{44}$. In this study, the masks were also $32 \times 32$-pixel, with the same resolution as that in the previous work. The continuously rotating disk was used in combination with photoconductive antennas as pulsed $\mathrm{THz}$ emitter and receiver. By fixing the delay line at the $\mathrm{THz}$ pulse electric field peak position and employing a compression factor (defined as the ratio between the number of patterns used to effectively reconstruct the image and the total number of basis patterns, i.e. total number of pixels) down to $16 \%$, an acquisition speed of $80 \mathrm{~s}$ per an image was achieved. Metallic masks were also used in $\operatorname{Ref}^{45}$, where the authors applied single-pixel imaging to $\mathrm{THz}$ light generated by a parametric oscillator. A Golay cell was used for $\mathrm{THz}$ power detection. In this case the image of a metallic $\mathrm{H}$-shaped object was retrieved multiple times, using different $\mathrm{THz}$ frequencies ranging from 1 to $2.2 \mathrm{THz}$. The authors compared the SNR of the image at different 


\section{a}

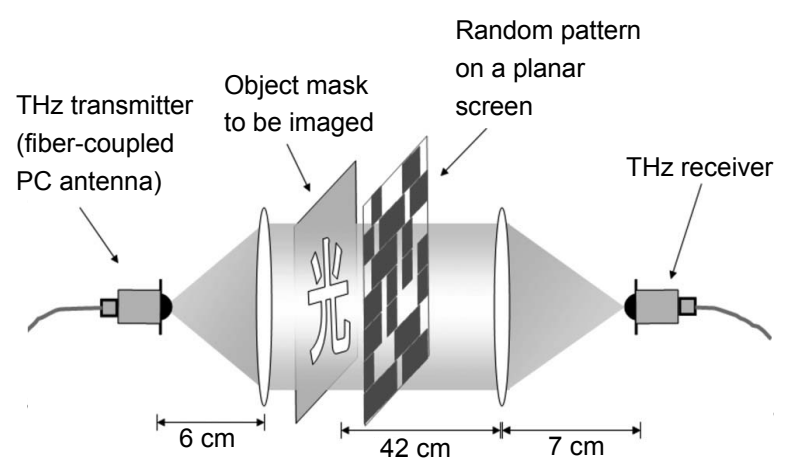

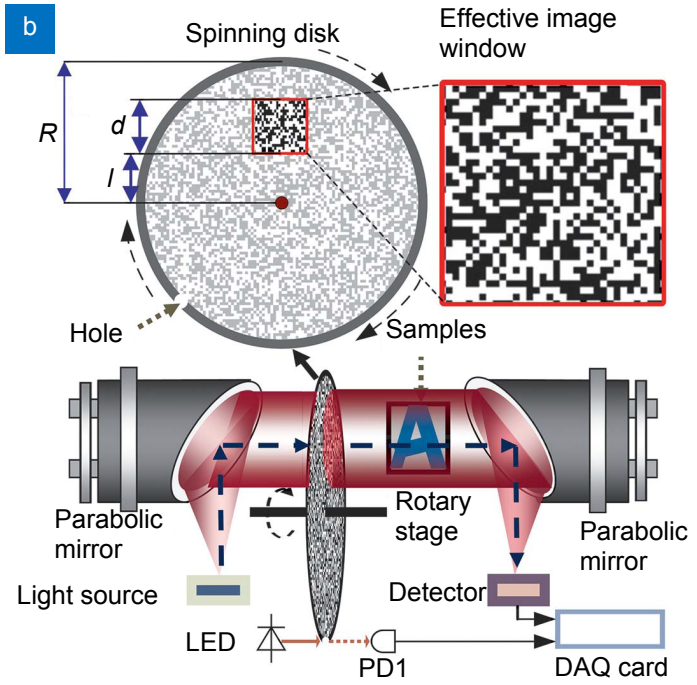

Fig. 2 | (a) Single-pixel imaging system based on metallic binary masks for THz modulation ${ }^{43}$. (b) Spinning-disk based THz imaging setup. Figure reproduced from: (a) Ref. ${ }^{43}$, AIP Publishing; (b) Ref. ${ }^{44}$, Optical Society of America.

frequencies, concluding that the best performance for the specific system employed was achieved at around 1.7 $\mathrm{THz}$ (SNR $=9 \mathrm{~dB}$, in correspondence to the maximum $\mathrm{THz}$ pulse energy of the source).

\section{All-optical free-carrier-based modulators}

The modulation technique that has become the most common in the last years is the one based on carrier photo-excitation in semiconductors. As shown in Fig. 3, a visible/near-infrared beam (typically obtained as a fraction of the pump beam used for $\mathrm{THz}$ light generation) is first patterned by means of a standard SLM, and then shined onto the surface of a semiconductor (often undoped silicon). The above-bandgap excitation generates free carriers in the illuminated regions. In "dark" conditions, the employed semiconductor is transparent to $\mathrm{THz}$ radiation, but in the photo-excited areas it behaves like a Drude metal, and this results in the absorption/reflection of the incident $\mathrm{THz}$ pulse. In this way, the spatial shape of the pattern is transferred from the optical beam to the $\mathrm{THz}$ beam. A comprehensive study on the main material parameters to consider for effectively applying photo-induced modulation to $\mathrm{THz}$ light can be found in Ref. ${ }^{46}$. Silicon, germanium and gallium arsenide were evaluated as modulating semiconductors. Tests were conducted for different semiconductor thicknesses and under variable illuminating power and wavelength. The features of the photo-carrier distribution in such conditions were analyzed.
The very first demonstration of such optical modulation technique is reported in Ref. ${ }^{47}$, where Q. Chen et al. used it as a mean to perform near-field imaging. The authors modulated only the center of the focused spot of a $\mathrm{THz}$ beam, directly on the surface of a sample made of GaAs, in order to increase the spatial resolution beyond the diffraction limit imposed by the long wavelength of $\mathrm{THz}$ radiation. The same technique was then employed, for the first time, for $\mathrm{THz}$ beam steering and imaging ${ }^{48}$, using pulsed $\mathrm{THz}$ radiation from a photoconductive antenna and incoherent light from a projector for photo-excitation. A $64 \times 64$-pixel image of a metallic cross was thus obtained by using a series of masks, each of them with only one pixel illuminated. The

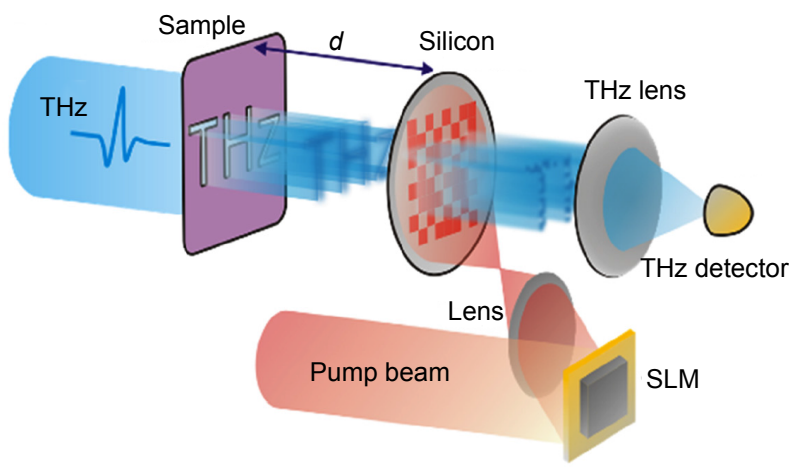

Fig. 3 | All-optical modulation of a THz beam. The patterning pump beam (visible/infrared) is modulated by an SLM. Subsequently, it illuminates the surface of a silicon plate and generates photo-carriers which transfer the pattern to the $\mathrm{THz}$ beam. Figure reproduced from Ref. ${ }^{52}$, Optical Society of America. 
first application exploiting multiplexed masks for single-pixel imaging appears in Ref. ${ }^{49}$. The authors used an arc lamp for $\mathrm{THz}$ generation and a bolometer for its detection, while the photo-exciting light came from a CW laser diode, emitting at $980 \mathrm{~nm}$. Images of metallic objects were acquired with increasing resolution, showing the superiority of multiplexed masks with respect to masks with a single illuminated pixel. Multiplexed masks allowed reasonable SNR conditions to be maintained, even with increased resolution. Though, no compression algorithm was applied, the flexibility and relative ease of implementation of this modulating technique have attracted the interest of the scientific community and various studies have explored different solutions to further improve it. In Ref. ${ }^{50}$, a modulation depth (the ratio of the transmitted $\mathrm{THz}$ powers at $100 \%$ and $0 \%$ of modulating light illumination) of $20 \mathrm{~dB}$ was achieved with incoherent white-light illumination from a projector. This is a convenient arrangement since it avoids the need of an amplified laser, but has, in general, limitations in the effective pattern resolution, due to carriers drifting within the semiconductor ${ }^{46}$. Such technique was used to spatially map the front of the $\mathrm{THz}$ beam and also showed the possibility of tracking moving objects. S. Augustin et al. ${ }^{51}$ employed a liquid crystal-based SLM to impress the pattern shapes onto the optical beam coming from a halogen lamp. Silicon was substituted with germanium for photo-modulation, since the lower bandgap of the latter allowed the use of less photo-modulating power. With this setup, the authors investigated the performance of a CS algorithm in the $\mathrm{THz}$ image reconstruction of a metallic object, showing well-retrieved images with a compression factor down to $30 \%$. In a recent paper ${ }^{52}$, Inverse Fresnel Diffraction (IFD) was also proposed to reconstruct the compressed imaging of a metallic object. The aim of this study, targeting far-field imaging implementations, was to show how the IFD allows correcting the effect of diffraction when the patterned $\mathrm{THz}$ beam propagates for a certain distance before interrogating the sample. Another recent work ${ }^{53}$ demonstrated the possibility of realizing and using "Fourier pattern" sets at $\mathrm{THz}$ frequencies. In this study, a silicon plate covered with a graphene monolayer was used as a photo-modulator, while the patterning source was a CW laser. In order to obtain Fourier patterns, 8-bit gray-scale masks (i.e., masks where the intensity in each pixel can assume 256 different values, instead of just the 2 values of basic binary masks) were encoded on the laser beam and projected onto the silicon plate. Since the density of the generated photo-carriers varies with the local laser intensity, the $\mathrm{THz}$ beam was also modulated in 8-bit patterns. CS reconstruction was then demonstrated with a compression factor down to $1.6 \%$ and reasonable SNR. Finally, in Ref. ${ }^{54}$, a novel technique for efficient $\mathrm{THz}$ modulation, based on $\mathrm{THz}$ total internal reflection (TIR) in a silicon prism, was presented. The modulation was in this case obtained by illuminating one face of the prism with a CW source, thus altering the total internal reflection condition for the $\mathrm{THz}$ beam (which entered the prism from one of its other faces). By using an SLM featuring a high switching rate to modulate the $\mathrm{CW}$ source and a fast TDS system based on PCAs, the authors reconstructed $32 \times 32$-pixel $\mathrm{THz}$ field-amplitude images at a notable rate of 6 frames $/ \mathrm{sec}$.

\section{THz SLMs}

Some implementations of $\mathrm{THz}$ SLMs have also been proposed in the last years. In Ref. ${ }^{55}$, a metamaterial-based SLM was used to reconstruct a 64-pixel image of a metallic cross (Fig. 4(a)), using an arc lamp as the $\mathrm{THz}$ source and a bolometer for detection. Each of the 64 pixels (Fig. 4(b)) of the SLM is constituted of an $18 \times 18$ array of metamaterial absorbers (MMAs) cells. These cells are designed to provide an artificial electromagnetic response that leads to an almost unitary absorption (zero reflection and transmission) at a specific frequency. By applying a reverse bias voltage, it is possible to tune the carrier concentration in a dielectric layer underneath the MMAs, thus varying both the real and imaginary part of the dielectric function. In this way, an electrically-controlled modulation of the absorption can be achieved in real-time (Fig. 4(d)). Furthermore, the response of the material can be frequency-tuned, potentially enabling narrow-band or hyperspectral imaging. In Ref. ${ }^{56}$ the authors show the fabrication and characterization of an SLM based on an array of 768 micro-mirrors, operating in the range $0.97-2.28 \mathrm{THz}$. The lateral size of the mirrors is of several hundred micrometers, in order to be compatible with diffraction at $\mathrm{THz}$ wavelengths. Although $\mathrm{THz}$ SLMs are currently a promising and active field of research, all-optical modulation still represents the most widespread and used method for single-pixel $\mathrm{THz}$ imaging, thanks to its flexibility, ease of implementation, and efficiency. 

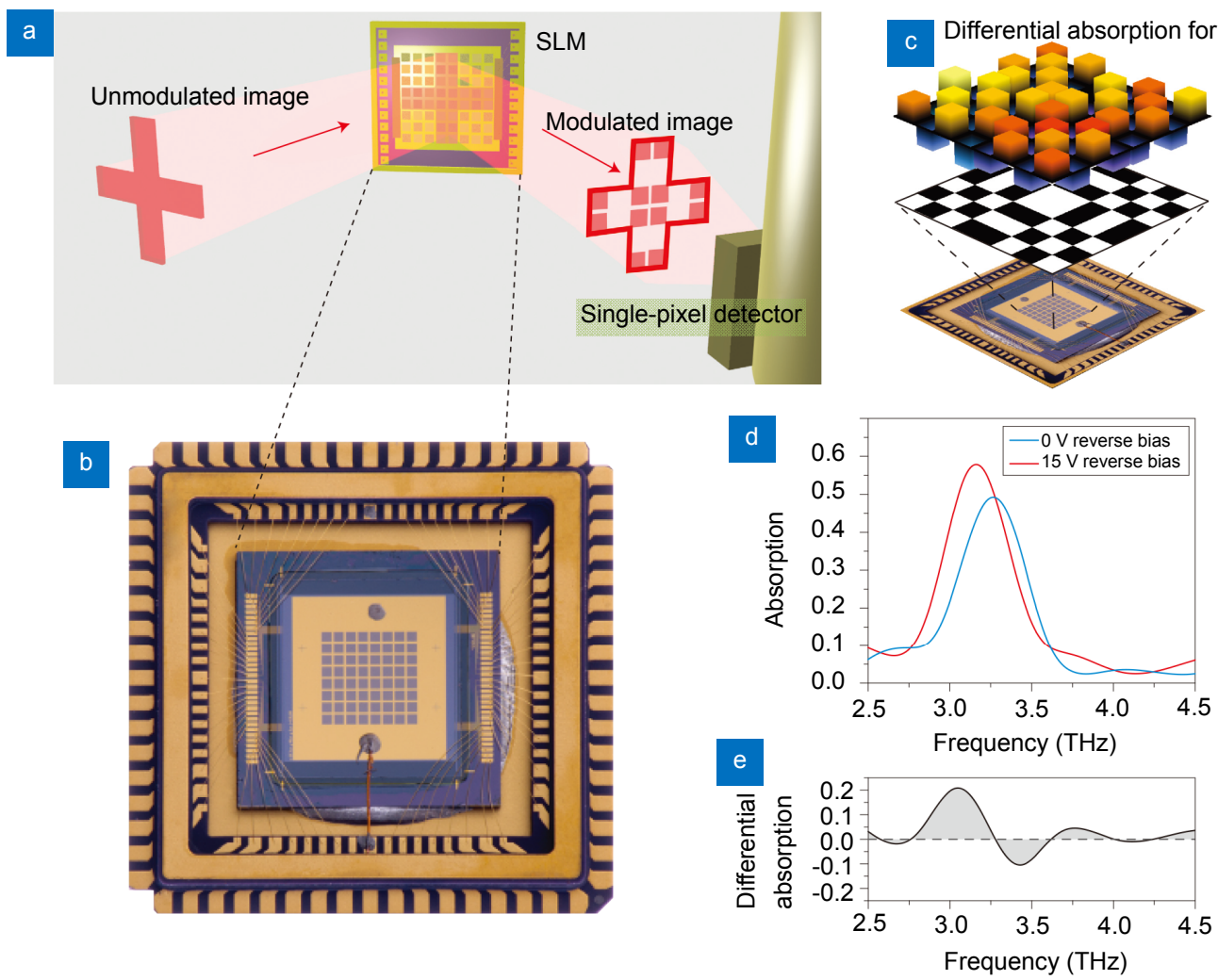

Fig. 4 | Metamaterial-based SLM ${ }^{55}$. (a) Operating principle of the SLM: THz light directly hits the SLM and the reflected beam results shaped into the spatial patterns. (b) Photograph of the SLM. (c) Map of the differential absorption for a +1/-1 Hadamard mask. (d) Absorption of the MMA cell for two different bias conditions $(0 \mathrm{~V}$ and $15 \mathrm{~V}$ ). As can be seen, amplitude and frequency of the absorption peak are different. (e) Differential absorption as a function of frequency. Figure reproduced from Ref. ${ }^{55}$, Springer Nature Limited.

\section{Patterning of the optical beam used for the generation/detection of $\mathrm{THz}$ radiation}

Alternative solutions have been proposed to spatially modulate the $\mathrm{THz}$ beam during the generation or detection processes. In Ref..$^{57}$, a collimated probe beam was used to sample the collimated $\mathrm{THz}$ beam in a $\mathrm{ZnTe}$ crystal via electro-optic sampling (as shown in Fig. 5(a)). The probe beam was previously patterned with the masks required for imaging, by means of a standard SLM. In this way, the $\mathrm{THz}$ beam was directly probed with the spatial patterns, after it had interacted with the object. The authors made use of two different series of patterns, Hadamard and random. They obtained good CS reconstruction with a compression factor down to $50 \%$. They also achieved subwavelength resolution of $\sim 60 \mu \mathrm{m}$, by placing the object at a distance of only $70 \mu \mathrm{m}$ from the detection crystal. Another technique, first proposed in a theoretical study ${ }^{58}$ and then implemented experimentally ${ }^{59}$, exploited a spatially-modulated pump beam for $\mathrm{THz}$ generation via optical rectification in a $\mathrm{ZnTe}$ crystal. In this way, the wave front of the $\mathrm{THz}$ pulse was directly shaped in space with the patterns during the nonlinear generation process (Fig. 5(b)). These two latter works considered the advantage of such a time-resolved non-linear ghost imaging (TNGI) in controlling the propagation of the patterned $\mathrm{THz}$ beam, in order to exploit the technique for near-field imaging. In particular, a strong spatiotemporal coupling effect was studied, which has significant implications in near-field time-domain imaging. Indeed, in the case of extremely localized (deep subwavelength) sources, the $\mathrm{THz}$ field spatial and temporal coordinates are coupled along propagation and the typical image reconstruction performed at fixed-time slices of the transmitted field becomes severely affected by errors and artifacts. Nevertheless, thanks to the capability of recording the electric field waveform in time offered by THz-TDS systems, such spatiotemporal effects can be successfully taken into account, thus enabling the faithful reconstruction of images in both the temporal and spatial domains with subwavelength resolution.

We conclude this section underlining the importance of $\mathrm{THz}$ modulation for the appropriate implementation of the single-pixel approach for $\mathrm{THz}$ imaging. All-optical 

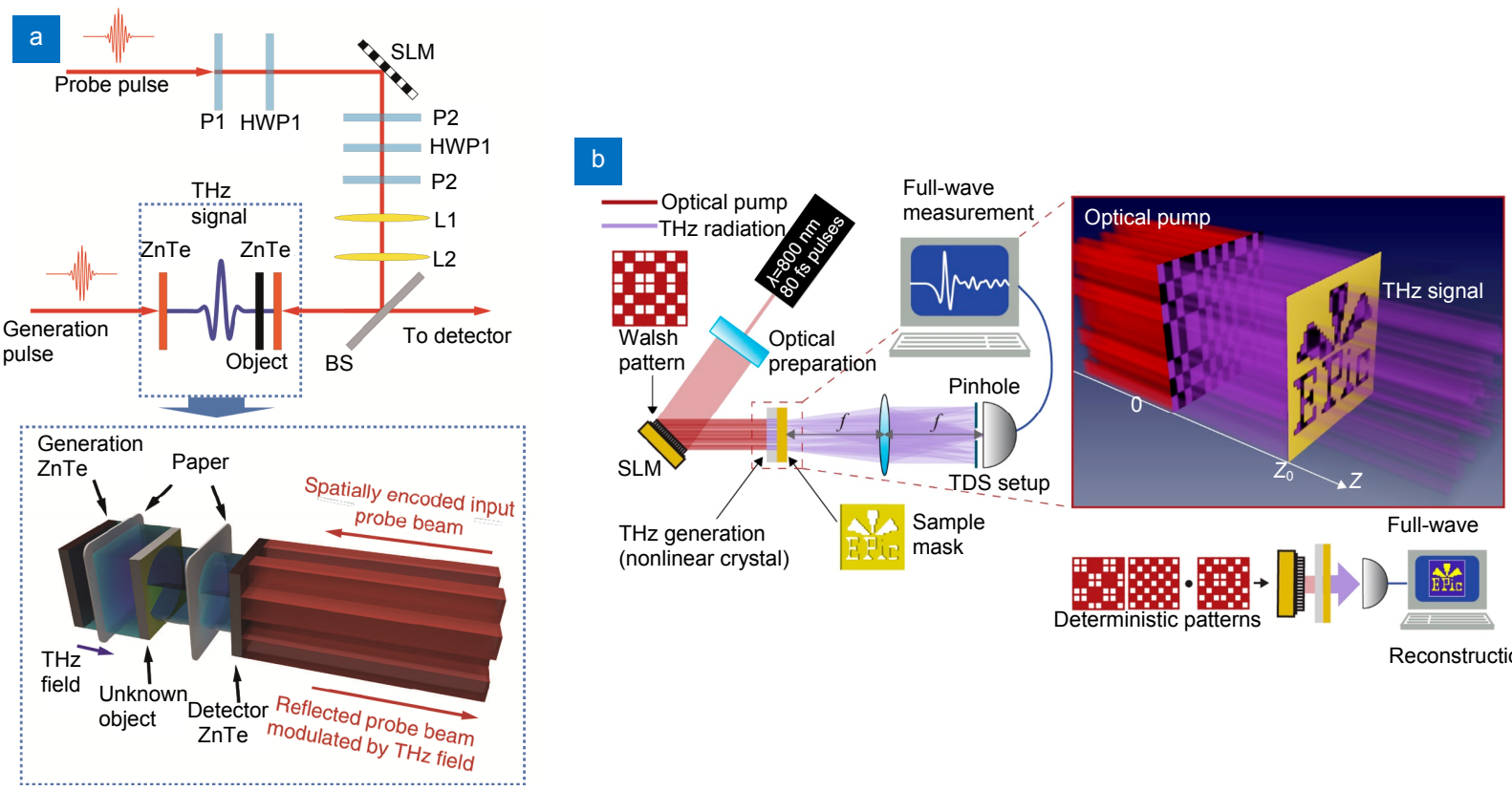

Reconstruction

Fig. 5 | (a) Experimental setup exploiting the patterning of the probe beam for $\mathrm{THz}$ imaging ${ }^{57}$. The spatial shape of the pattern is encoded via an SLM on an optical beam, which is used to probe the collimated THz beam in the detection crystal. (b) Experimental implementation of TNGI ${ }^{58}$. In this case, the pump beam generating $\mathrm{THz}$ radiation is spatially modulated, allowing to obtain a $\mathrm{THz}$ beam already shaped into the required masks. Figure reproduced from: (a) Ref. ${ }^{57}$, Creative Common 4.0 International License; (b) Ref. ${ }^{58}$, American Chemical Society.

techniques based on free-carrier generation are the ones that so far have received the largest attention, thanks to their flexibility, and have thus been developed and applied the most. Nevertheless, THz SLMs and other alternative methods have also been recently investigated and they are likely to become more commonly used in the years to come.

\section{Examples of applications}

In this section, we will consider works applying the single-pixel configuration to some typical context where $\mathrm{THz}$ imaging is used. Such investigations consist mainly of proof-of-concept experiments that are relevant to prove the effectiveness of the technique in view of future practical implementations. We divide them in far-field, near-field, and alternative imaging schemes, due to the intrinsic difference in the working conditions as well as potential applications.

\section{Far-field phase-contrast, hyperspectral, and time-domain imaging}

In the first application of single-pixel imaging at $\mathrm{THz}$ frequencies with metallic masks ${ }^{43}$, Chan et al. demonstrated the possibility of reconstructing images in the far-field, both with spectral amplitude measurements, and with phase contrast ones. In the amplitude-only case, they used a metallic object, opaque to $\mathrm{THz}$ radiation, and showed CS reconstruction of a 1024-pixel image $(32 \times 32)$ using only 300 patterns. In the second case, they obtained the image of a rectangle of Teflon (transparent to $\mathrm{THz}$ radiation) using a phase contrast technique. They applied a reconstruction algorithm able to retrieve both the amplitude and the phase associated to single frequencies contained in a broadband THz pulse. The 1024-pixel image was reconstructed with 400 phase measurements, at the specific frequency of $0.1 \mathrm{THz}$. In Ref. ${ }^{60}$, a phase-sensitive implementation with intensity-only measurements was presented, based on two vector network analyzers (VNA) coupled with horn antennas for $\mathrm{THz}$ generation/detection, and a silicon-based optical modulator. For the retrieval of phase contrast images, an algorithm called Phaselift was used. The crucial point of this experiment was the ability of applying a series of complex masks modulating, at the same time, both the intensity amplitude and phase associated with a specific frequency component $(690 \mathrm{GHz})$. This is possible since the layer of carriers, generated on the silicon surface, can induce a phase shift, in addition to the well-known amplitude modulation, due to the change of the complex refractive index of the material. These first two works presented two different ways to enable phase-contrast imaging. Another powerful capability of $\mathrm{THz}$ imaging is the spectral recognition of substances. In Ref. ${ }^{61}$, the authors presented the first use of single-pixel imaging for 
spectroscopic recognition. A composite material made of polyethylene and lactose was analyzed. By considering the difference in absorption at specific frequencies in the spectrum (Fig. 6(a)), it was possible to identify the regions where the lactose (featuring two main absorption lines at $0.54 \mathrm{THz}$ and $1.38 \mathrm{THz}$ ) was present (Fig. 6(b)). Even in this case CS was applied, enabling image reconstruction with only $10 \%$ of the total masks. The abovementioned works present $\mathrm{THz}$ imaging results either with phase retrieval at individual frequencies, or with differential amplitude reconstruction for spectral recognition. In a recently published investigation ${ }^{62}$, the complete reconstruction of few-cycle $\mathrm{THz}$ pulses directly in the time domain at each pixel position was achieved, starting from the waveforms recorded in time for each of the patterns (Fig. 6(c)). It was demonstrated that the single-pixel imaging paradigm is able to provide the same amount of information one would obtain by raster-scanning the object in a standard THz TDS-based implementation. Such temporal reconstruction was obtained operating in CS conditions (using down to $40 \%$ of the total masks), thus reducing the acquisition time. As a proof of principle, the technique was applied to two common situations where $\mathrm{THz}$ imaging is used, namely time-of-flight imag- ing and spectroscopic recognition. For the time-of-flight test, a sample made of high-density polyethylene (HDPE) with variable thickness was employed (Fig. 6(d)), and "time delay" $16 \times 16$-pixel images were successfully reconstructed (Fig. 6(e)). For what concerns spectroscopic recognition, $8 \times 8$-pixel spectral images of a sample made of a mixture of Teflon and lactose were obtained by direct Fourier transformation of the $\mathrm{THz}$ temporal waveform retrieved in each pixel.

All these studies have been pursuing the same general objective, i.e. multidimensional far-field imaging at $\mathrm{THz}$ frequencies with a reduced acquisition time. This means obtaining additional information from the object under investigation, being it in the spectral or temporal domain, beyond its spatial characteristics, thus fully harvesting the most powerful peculiarities of traditional $\mathrm{THz}$ imaging.

\section{Near-field imaging}

Significant efforts have been devoted in the last years to applying the single-pixel approach to $\mathrm{THz}$ near-field imaging, in order to improve the achievable spatial resolution. Indeed, the long wavelength of $\mathrm{THz}$ radiation typically limits the resolution in the far-field to hundreds of microns. A series of publications by Stantchev et al.
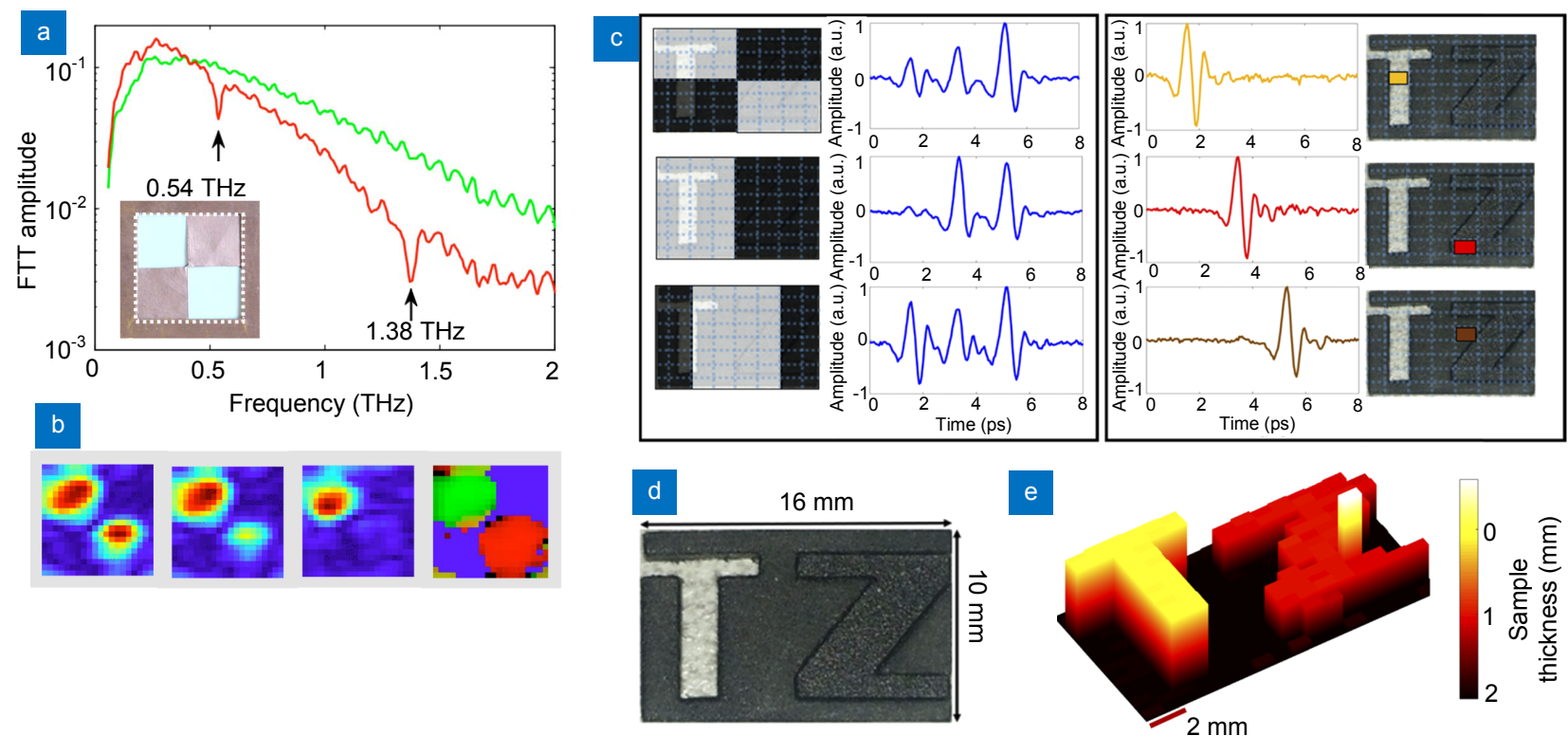

Fig. 6 | (a) THz spectra of polyethylene (upper trace) and lactose (lower) and photo of the sample (polyethylene at the upper-left side, lactose at the bottom-right and copper in the rest of the surface ${ }^{61}$.(b) Spectral images of the sample shown in the inset of (a): the images (left to right) are reconstructed considering the absorption at $0.50 \mathrm{THz}, 0.54 \mathrm{THz}, 1.38 \mathrm{THz}$, respectively, while the last is an RGB map of the regions with different absorption features (lactose in red, polyethylene in green, copper in blue). The maps were obtained by Fourier-transforming the THz waveforms measured for each spatial pattern and then reconstructing the spectral amplitude in each pixel for the 3 frequencies of interest. (c) Time-domain reconstruction of $\mathrm{THz}$ waveforms ${ }^{62}$ : On the left side, the waveforms recorded for some of patterns; on the right side, those reconstructed in some selected pixels. (d) HDPE sample used in Ref. ${ }^{62}$. (e) $3 \mathrm{D}$ image of the sample in (d), retrieved using the relative time delays of the THz pulses at each pixel, showing three different thicknesses. Figure reproduced from: (a, b) Ref. ${ }^{61}$, AlP Publishing; (c-e) Ref. ${ }^{62}$, Optical Society of America. 


\section{Opto-Electronic Advances https://doi.org/10.29026/oea.2020.200012}

studied different aspects and applications of near-field $\mathrm{THz}$ imaging. In Ref. ${ }^{63}$, the reconstruction of the image of a metallic cartwheel and a circuit board was reported, achieving resolutions down to $100 \mu \mathrm{m}(\sim \lambda / 4)$. The authors reported that the key limitation preventing a further improvement of the resolution lied within the thickness of the employed silicon-based modulator, which was in this case in direct contact with the object for near-field imaging purposes. Moreover, two different sets of patterns, Hadamard and random, were applied, showing the superiority of Hadamard masks to obtain a high spatial resolution. In a subsequent study ${ }^{64}$, the image of the same cartwheel was obtained with a $9 \mu \mathrm{m}$ resolution, as shown in Fig. $7(\mathrm{c} 1)$. In this case, the photo-modulation technique was exploited in thin silicon wafers (with thickness down to $6 \mu \mathrm{m}$ ) supporting the object to be imaged. Interestingly, the resolution was affected by the polarization of the $\mathrm{THz}$ beam, with a lower resolution in the regions where the polarization was almost parallel to the edges of the cartwheel, due to different electromagnetic boundary conditions at the metal air interface. In Ref. ${ }^{65}$, the same group investigated the conductivity of a graphene monolayer, by depositing it on the surface of the silicon modulator. Conductivity maps with a resolution down to $75 \mu \mathrm{m}$ were obtained. In Ref. ${ }^{66}$, a hyperspectral study of a cartilage sample with subwavelength thickness was performed with a similar setup. The permittivity of the cartilage was extracted, correlating its spatial variation to the change of the collagen fibril orientation within the sample. Additional work was then performed to improve the spatial resolution even further. In Ref. ${ }^{67}$, Chen et al. achieved resolutions down to $\lambda / 100(4.5 \mu \mathrm{m})$ while imaging a metallic object. This was made possible by using a thin film of vanadium dioxide $\left(\mathrm{VO}_{2}\right)$ with a thickness of only $180 \mathrm{~nm}$ for $\mathrm{THz}$ spatial modulation. Besides improving the spatial resolution, another crucial challenge in $\mathrm{THz}$ near-field single-pixel imaging is represented by the time-domain $\mathrm{THz}$ waveform reconstruction, which enables phase and spectral imaging. A very recent study by Olivieri et al..$^{59}$, demonstrated the reconstruction of the $\mathrm{THz}$ waveform in the time-domain, for each pixel of subwavelength lateral size (down to $125 \mu \mathrm{m}$ ), while imaging a leaf (Figs. $7(\mathrm{a} 2)-7(\mathrm{j} 2)$ ). In this case, the authors performed single-pixel near-field imaging by directly patterning the optical beam pumping the $\mathrm{THz}$ generation
Experiment

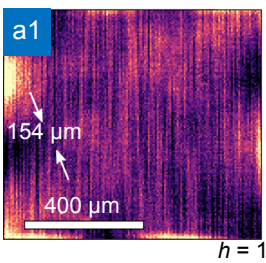
$h=400 \mu \mathrm{m}$

\section{Modeling}
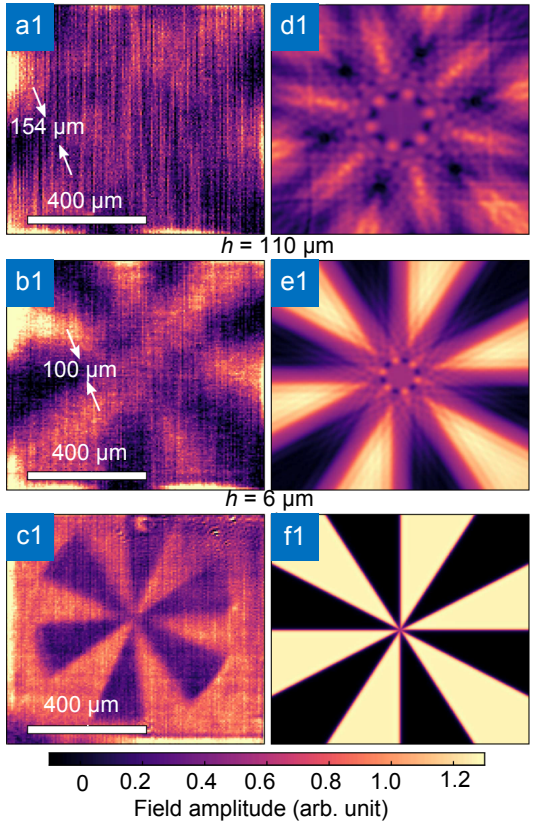
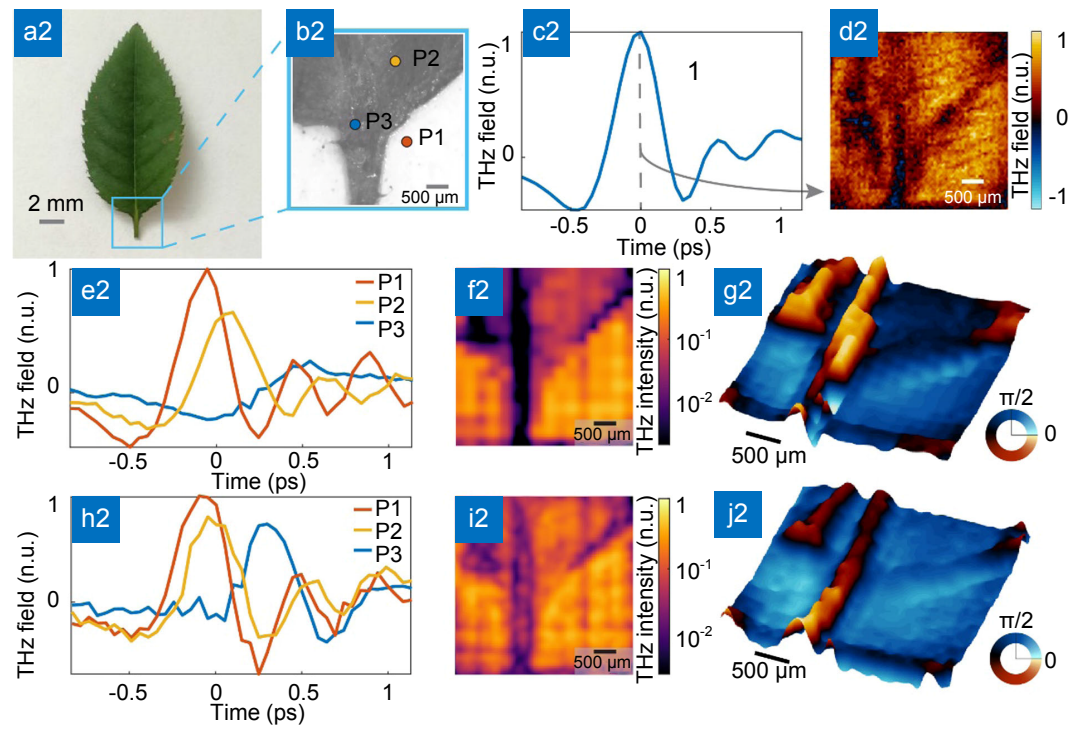

Fig. 7 | (a1-f1) THz near-field imaging of a metallic cartwheel ${ }^{64}$. The images present the comparison between the experimental results and numerical modeling, for silicon modulators with decreasing thickness (400 $\mu \mathrm{m}$ (a1), (d1), $110 \mu \mathrm{m}$ (b1), (e1), $6 \mu \mathrm{m}$ (c1), (f1)). As can be clearly seen, only the $6 \mu \mathrm{m}$-thick silicon wafer allows to resolve the central region with smaller features. (a2-j2) Hyperspectral image of a leaf ${ }^{59}$ : (a2) Optical image of the leaf; (b2) microscope image; (c2) temporal waveform of the THz field transmitted through the leaf; (d2) fixed-time reconstruction (128 $\times 128$-pixels); (e2) local temporal response of the fresh leaf in the points indicated in (b2); (f2) hyperspectral image of a fresh leaf at $1.5 \mathrm{THz}$ (16 $\times$ 16 -pixels); (g2) phase image of the fresh leaf, obtained without phase unwrapping of the experimental data; (h2-j2) same as the previous panel for a dried leaf $\left(32 \times 32\right.$-pixels image). All the images correspond to a field of view of $4 \mathrm{~mm} \times 4 \mathrm{~mm}$. Figure reproduced from: (a1-f1) Ref. ${ }^{64}$, The Optical Society; (a2-j2) Ref. ${ }^{59}$, Optical Society of America. 


\section{Opto-Electronic Advances https://doi.org/10.29026/oea.2020.200012}

crystal (TNGI, see previous section). The theoretical framework developed to describe these conditions ${ }^{58}$ clearly evidenced that spatiotemporal coupling effects have to be properly considered to reconstruct the image when the pixels are of sub-wavelength dimensions. The authors thus provided a method (space-time refocusing) to achieve such reconstruction. The technique was also successfully applied to a metallic sample placed at a distance of hundreds of microns from the position where the patterns were generated. It is worth stressing that a crucial aspect of the developed technique is represented by the need of recording the full $\mathrm{THz}$ waveform in the time domain.

\section{Alternative single-pixel THz imaging implementations}

Aside from the research lines reported in the two previous sub-sections, other interesting studies have very recently explored different implementations and applications of single-pixel $\mathrm{THz}$ imaging. In Ref. ${ }^{68}$, single-pixel imaging was applied to a two-dimensional $\mathrm{THz}$ tomographic system. For this type of imaging, multiple images at different angles are captured in transmission. Subsequently, a reconstruction algorithm is used to combine the images, thus retrieving the inner features of the object. Mohr et al. acquired each of these images by using a single-pixel imaging configuration. Interestingly, the experimental setup made use of a commercial Fourier-transform spectrometer, with an arc lamp as the $\mathrm{THz}$ source and a bolometer for $\mathrm{THz}$ detection, while the modulation of the $\mathrm{THz}$ beam was achieved optically, by illuminating a silicon plate with a beam from a laser diode, previously patterned by means of a micro-mirror device. Two polypropylene objects with cylindrical and cuboid (Figs. 8(a)-8(d)) shapes were imaged. The authors also developed a model to account for distortions of the pattern shape due to reflection, refraction and diffraction, obtaining good agreement between the model predictions and the experimental results. Another recent work ${ }^{69}$ made use of a single-bit sensor (i.e., a detector featuring only two different value states, 0 and 1) for implementation into a single-pixel $\mathrm{THz}$ imaging system. The authors compared the intensity measurement of a given mask with its complementary version. By considering only the "sign" (positive or negative) of the difference between the sensor readings for complementary masks, a compression value down to $25 \%$ was achieved. Moreover, the improved performance of the system when compared to regular CS in conditions of low SNR was demonstrated. The same group also
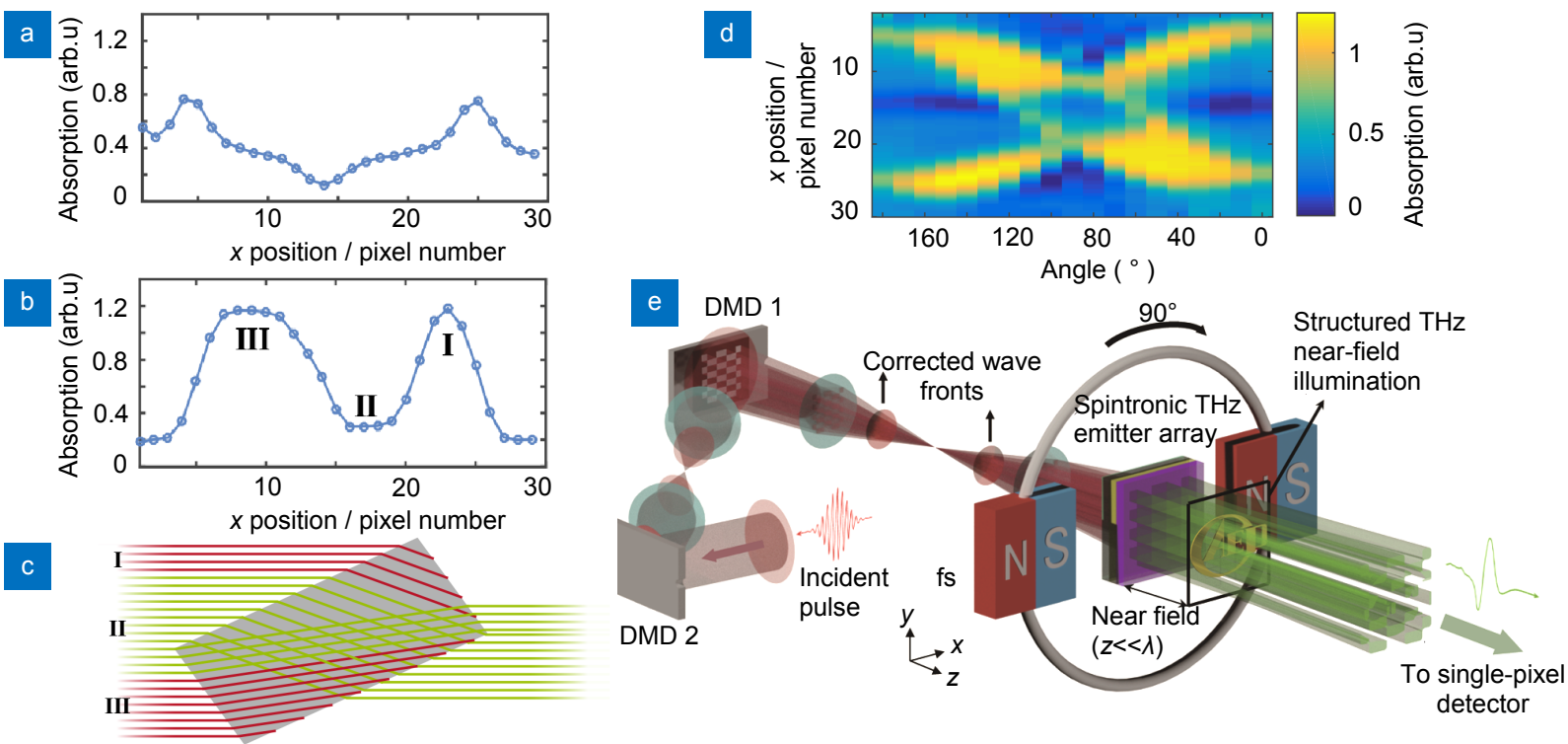

Fig. 8 | (a-d) Tomographic reconstruction of a rectangular object ${ }^{68}$ : two measured projections under a rotation angle of (a) $180^{\circ}$ and (b) $120^{\circ}$ (c) Ray tracing simulation for a rectangular object with edge lengths of $14 \mathrm{~mm} \times 7 \mathrm{~mm}$ and a refractive index of 1.5 under a rotation of $120^{\circ}$. Red colored rays indicate rays which get totally reflected on the rear side, while green rays can pass through the object. (d) Measured sinogram of a cuboid polypropylene sample with edge lengths of $14 \mathrm{~mm} \times 7 \mathrm{~mm}$. (e) Schematic of the GHOSTEAM system ${ }^{71}$ : the spintronic THz emitter array (STEA) is excited by two-DMDs-encoded fs-laser pulses and generates spatially coded THz pulses. An object is placed in the near-field region $(z<<\lambda)$. The transmitted THz pulse is collected and sent to a single-pixel detector. Figure reproduced from: (a-d) Ref. ${ }^{68}$, Optical Society of America; (e) Ref. ${ }^{71}$, Creative Common 4.0 International License. 
presented another $\mathrm{THz}$ compressive imaging approach, by employing multiple sensors ${ }^{70}$. The idea underlying this work is to illuminate the sample with patterned light, as in the case of traditional single-pixel imaging, and then record the $\mathrm{THz}$ intensity in multiple selected points of the Fourier space (specifically, the technique was proven using two points). By employing such technique, the number of required masks (and consequently the acquisition time) decreases linearly with the increase in the number of sensors, without any degradation of the quality of the reconstruction in comparison with the traditional single-pixel scheme. In Ref. ${ }^{71}$, Chen et al. introduced the use of an innovative system enabling ultrabroadband $\mathrm{THz}$ generation, i.e. a spintronic $\mathrm{THz}$ emitter array (STEA), for single-pixel imaging (Fig. 8(e)). Spintronic $\mathrm{THz}$ emitters, exploiting the ferromagnetic properties of thin metal films, have been recently demonstrated $^{72}$. In these devices, a layer of a ferromagnetic material is combined with a non-ferromagnetic film. A femtosecond laser excites the former, generating a current that is spin-polarized. When this current moves to the non-ferromagnetic layer, it undergoes a deflection due to an inverse spin-Hall effect, resulting in an ultrafast transverse current density, which acts as a $\mathrm{THz}$ source. The polarization of the emitted $\mathrm{THz}$ pulses can be controlled via an external magnetic field. One of the advantages of this generation technique is the gapless and wide frequency-band readily available $(0.3-30 \mathrm{THz})$, thanks to the absence of detrimental optical phonon modes ${ }^{72}$, which instead hampers the performance of typical semiconductor-based $\mathrm{THz}$ sources. However, it should be noted that, in Ref. ${ }^{71}$, the detection of the $\mathrm{THz}$ pulses was still performed via electro-optic sampling in a 1-mm-thick ZnTe crystal, thus limiting the available bandwidth to less than $2 \mathrm{THz}$. In the STEA ${ }^{71}$, every element acts as an individual $\mathrm{THz}$ emitter, which is independently programmable by photoexcitation. The masks, impressed on the pump laser beam with an SLM, illuminate the STEA, where they selectively excite every element and transfer the pattern to the $\mathrm{THz}$ beam. This generation technique was tested for near-field applications, exploiting the fact that the elements of the array had a small size of $6.5 \mu \mathrm{m} \times 6.5 \mu \mathrm{m}$, and the sample could be placed at only $150 \mathrm{~nm}$ from the source (corresponding to the thickness of a protective $\mathrm{SiO}_{2}$ layer). Thus, using this experimental system, super-resolution was achieved. Furthermore, the ability of reconstructing $\mathrm{THz}$ amplitude images at different fixed time delays was also shown.

\section{Conclusions}

In conclusion, we have presented an overview of the state of the art regarding the use of the single-pixel approach in $\mathrm{THz}$ imaging systems. We have briefly introduced the key motivations underlying these studies and subsequently described the concept of single-pixel imaging, listing its peculiarities and advantages, in particular for applications at $\mathrm{THz}$ frequencies. We have then reported the experimental progress achieved so far. We have first discussed an important experimental challenge, which concerns $\mathrm{THz}$ spatial modulation, and listed the solutions proposed to address it. Initially, physical metallic masks were employed, which needed to be mechanically displaced. Subsequently, an approach based on photo-modulation in semiconductors was developed. This latter technique has become the most commonly employed, thanks to its flexibility and ease of implementation, and has been improved over the years. Other interesting solutions were explored lately, ranging from the development of $\mathrm{THz}$ SLMs, to $\mathrm{THz}$ generation/detection with patterned light. We have then proceeded to the description of some proof-of-principle uses of single-pixel imaging for typical $\mathrm{THz}$ implementations. We have first considered far-field imaging applications, reporting on the successful demonstration of phase-contrast, hyper-spectral and time-domain $\mathrm{THz}$ imaging. This last achievement, in particular, enables the access to the full amount of information available from standard TDS-based imaging systems. Afterwards, we have shifted to near-field applications, the main purpose of which is to improve the spatial resolution of THz imaging. Near-field single-pixel $\mathrm{THz}$ imaging was first demonstrated with field amplitude measurements, thereafter for hyperspectral imaging, and ultimately, early this year, was also combined with the temporal reconstruction of the $\mathrm{THz}$ waveform. Finally, we have presented alternative approaches where the single-pixel strategy has been recently used. The unique abilities of $\mathrm{THz}$ radiation in investigating the properties of materials make it potentially attractive for a vast variety of industrial applications. Enabling fast (ideally, real-time) imaging at $\mathrm{THz}$ frequencies is therefore one of the crucial challenges to make $\mathrm{THz}$ technology appealing for large scale applications. Single-pixel solutions are among the most promising techniques to pursue this objective. They present advantages in terms of cost, speed, 
efficiency and ease of implementation. In the last decade, the interest of the scientific community on this topic has been growing steadily and new, progressively more effective systems have been explored. Single-pixel cameras are currently an active area of research for a very wide spectrum of uses beyond $\mathrm{THz}$ technology, in all those applications where traditional, silicon-based sensors show their limits. Such research efforts will likely be also beneficial for a prompt implementation of these devices in commercial $\mathrm{THz}$ systems. We believe that the development of $\mathrm{THz}$ technology is bound to expand over the next years, targeting a variety of applications in security, sensing, as well as manufacturing process monitoring in industrial production. Single-pixel imaging can represent one of the key solutions to make $\mathrm{THz}$ radiation more suitable for such practical uses. We foresee that research in this field will follow two main directions: i) further improving $\mathrm{THz}$ modulation technology, and ii) reducing the time required to retrieve the $\mathrm{THz}$ temporal waveforms. Regarding point i), a new generation of $\mathrm{THz}$ SLMs providing large modulation depths, high switching rates, low energy consumption at a low cost would be extremely appealing. For example, this would enable implementing simplified single-pixel imaging schemes in THz-TDS setups based on photo-conductive antennas and driven by affordable high-repetition rate, low-energy fiber-based laser oscillators, thus reducing the overall cost of the system while significantly boosting the SNR. As for point ii), advanced schemes such as the so-called "single-shot" detection techniques ${ }^{73}$ may be implemented to avoid lengthy $\mathrm{THz}$ waveform scans in time. The combination of the latter techniques with the single-pixel approach for image formation could ultimately allow reaching the goal of real-time $\mathrm{THz}$ multidimensional imaging.

\section{References}

1. Mittleman D M. Twenty years of terahertz imaging [Invited]. Opt Express 26, 9417-9431 (2018).

2. Mittleman $D \mathrm{M}$, Jacobsen $\mathrm{R} \mathrm{H}$, Nuss $\mathrm{M} \mathrm{C}$. T-ray imaging. IEEE J Sel Top Quantum Electron 2, 679-692 (1996).

3. Liu H B, Zhong H, Karpowicz N, Chen Y Q, Zhang X C. Terahertz spectroscopy and imaging for defense and security applications. Proc IEEE 95, 1514-1527 (2007).

4. Stoik C, Bohn M, Blackshire J. Nondestructive evaluation of aircraft composites using reflective terahertz time domain spectroscopy. NDT E Int 43, 106-115 (2010).

5. Blanchard F, Doi A, Tanaka T, Tanaka K. Real-time, subwavelength terahertz imaging. Annu Rev Mater Res 43, 237-259 (2013).

6. Jacobsen R H, Mittleman D M, Nuss M C. Chemical recognition of gases and gas mixtures with terahertz waves. Opt Lett 21 2011-2013 (1996).

7. Parrott E P J, Zeitler J A. Terahertz time-domain and low-frequency raman spectroscopy of organic materials. Appl Spectrosc 69, 1-25 (2015).

8. Baxter J B, Guglietta G W. Terahertz spectroscopy. Anal Chem 83, 4342-4368 (2011).

9. Jepsen P U, Cooke D G, Koch M. Terahertz spectroscopy and imaging-Modern techniques and applications. Laser Photon Rev 5, 124-166 (2011).

10. Shen Y C, Lo T, Taday P F, Cole B E, Tribe W R et al. Detection and identification of explosives using terahertz pulsed spectroscopic imaging. Appl Phys Lett 86, 241116 (2005).

11. Duling I, Zimdars D. Revealing hidden defects. Nat Photon 3, 630-632 (2009).

12. Jördens $C$, Koch $M$. Detection of foreign bodies in chocolate with pulsed terahertz spectroscopy. Opt Eng 47, 037003 (2008).

13. Jansen $\mathrm{C}$, Wietzke $\mathrm{S}$, Peters $\mathrm{O}$, Scheller $\mathrm{M}$, Vieweg $\mathrm{N}$ et al. Terahertz imaging: Applications and perspectives. Appl Opt 49, E48-E57 (2010).

14. Sun Q S, He Y Z, Liu K, Fan S T, Parrott E P J et al. Recent advances in terahertz technology for biomedical applications. Quant Imaging Med Surg 7, 345-355 (2017).

15. Yang $X$, Zhao X, Yang K, Liu Y P, Liu Y et al. Biomedical applications of terahertz spectroscopy and imaging. Trends Biotechnol 34, 810-824 (2016).

16. Naccache R, Mazhorova A, Clerici M, Piccoli R, Khorashad L K et al. Terahertz thermometry: combining hyperspectral imaging and temperature mapping at terahertz frequencies. Laser Photon Rev 11, 1600342 (2017).

17. Koch-Dandolo C L, Filtenborg T, Fukunaga K, Skou-Hansen J, Jepsen $\mathrm{P} U$. Reflection terahertz time-domain imaging for analysis of an 18th century neoclassical easel painting. Appl Opt 54, 5123-5129 (2015).

18. Abraham E, Younus A, Delagnes J C, Mounaix P. Non-invasive investigation of art paintings by terahertz imaging. Appl Phys $A$ 100, 585-590 (2010).

19. Dong J L, Locquet A, Melis M, Citrin D S. Global mapping of stratigraphy of an old-master painting using sparsity-based terahertz reflectometry. Sci Rep 7, 15098 (2017).

20. Menlo. THz Imaging Tool with ImageLab Processing Software. https://www.menlosystems.com/products/thz-time-domain-solut ions/tera-image-3/.

21. Guerboukha $\mathrm{H}$, Nallappan $\mathrm{K}$, Skorobogatiy $\mathrm{M}$. Toward real-time terahertz imaging. Adv Opt Photon 10, 843-938 (2018).

22. Lee A W M, Qin Q, Kumar S, Williams B S, Hu Q. Real-time terahertz imaging over a standoff distance (>25meters). Appl Phys Lett 89, 141125 (2006).

23. Nemoto $\mathrm{N}$, Kanda $\mathrm{N}$, Imai $\mathrm{R}$, Konishi $\mathrm{K}$, Miyoshi $\mathrm{M}$ et al. High-sensitivity and broadband, real-time terahertz camera incorporating a micro-bolometer array with resonant cavity structure. IEEE Trans Terahertz Sci Technol 6, 175-182 (2016).

24. Yang J, Ruan S C, Zhang M. Real-time, continuous-wave terahertz imaging by a pyroelectric camera. Chin Opt Lett 6, 29-31 (2008).

25. Al Hadi R, Sherry $H$, Grzyb J, Zhao $Y$, Förster $W$ et al. A 1 k-pixel video camera for 0.7-1.1 terahertz imaging applications in 65-nm CMOS. IEEE J Solid-State Circuits 47, 2999-3012 (2012).

26. Cooper K B, Dengler R J, Llombart N, Thomas B, 
Chattopadhyay $\mathrm{G}$ et al. THz imaging radar for standoff personnel screening. IEEE Trans Terahertz Sci Technol 1, 169-182 (2011).

27. Katletz S, Pfleger M, Pühringer $\mathrm{H}$, Vieweg N, Scherger B et al. Efficient terahertz en-face imaging. Opt Express 19, 23042 (2011).

28. Henry S C, Zurk L M, Schecklman S. Terahertz spectral imaging using correlation processing. IEEE Trans Terahertz Sci Technol 3, 486-493 (2013).

29. Ushakov A, Chizhov P, Bukin V, Savel'ev A, Garnov S. Broadband in-line terahertz 2D imaging: comparative study with time-of-flight, cross-correlation, and Fourier transform data processing. J Opt Soc Am B 35, 1159-1164 (2018).

30. Schumann S, Jansen C, Schwerdtfeger M, Busch S, Peters O et al. Spectrum to space transformed fast terahertz imaging. Opt Express 20, 19200-19205 (2012).

31. Guerboukha H, Nallappan K, Skorobogatiy M. Exploiting k-space/frequency duality toward real-time terahertz imaging. Optica 5, 109-116 (2018).

32. Edgar M P, Gibson G M, Padgett M J. Principles and prospects for single-pixel imaging. Nat Photon 13, 13-20 (2019).

33. Candes E J, Wakin M B. An introduction to compressive sampling. IEEE Signal Process Mag 25, 21-30 (2008).

34. M. (Center for R. and S. R. Harwit, N. Y. Cornell University, Ithaca, N. J. A. (Bell L. Sloan, and N. J. Murray Hill, Hadamard Transform Optics (Academic Press, 1979).

35. Zhang Z B, Ma X, Zhong J G. Single-pixel imaging by means of Fourier spectrum acquisition. Nat Commun 6, 6225 (2015).

36. Zhang Z B, Wang X Y, Zheng G A, Zhong J G. Hadamard single-pixel imaging versus Fourier single-pixel imaging. Opt Express 25, 19619-19639 (2017).

37. Stern A. Optical Compressive Imaging (CRC Press/Taylor \& Francis, 2016).

38. Duarte M F, Davenport M A, Takbar D, Laska J N, Sun T et al. Single-pixel imaging via compressive sampling: Building simpler, smaller, and less-expensive digital cameras. IEEE Signal Process Mag 25, 83-91 (2008).

39. Candes E J, Tao T. Near-optimal signal recovery from random projections: Universal encoding strategies? IEEE Trans Inf Theory 52, 5406-5425 (2006).

40. Donoho D L. Compressed sensing. IEEE Trans Inf Theory $\mathbf{5 2 ,}$ 1289-1306 (2006).

41. Candès E J, Romberg J, Tao T. Robust uncertainty principles: Exact signal reconstruction from highly incomplete frequency information. IEEE Trans Inf Theory 52, 489-509 (2006).

42. Augustin S, Frohmann S, Jung P, Hübers H W. Mask responses for single-pixel terahertz imaging. Sci Rep 8, 4886 (2018).

43. Chan W L, Charan K, Takhar D, Kelly K F, Baraniuk R G et al. A single-pixel terahertz imaging system based on compressed sensing. Appl Phys Lett 93, 121105 (2008).

44. Shen H, Gan L, Newman N, Dong Y, Li C et al. Spinning disk for compressive imaging. Opt Lett 37, 46-48 (2012).

45. Duan $P$, Wang $Y Y, X u$ D G, Yan C, Yang $Z$ et al. Single pixel imaging with tunable terahertz parametric oscillator. Appl Opt 55, 3670-3675 (2016).

46. Kannegulla A, Shams M I B, Liu L, Cheng L J. Photo-induced spatial modulation of $\mathrm{THz}$ waves: opportunities and limitations. Opt Express 23, 32098-32112 (2015).

47. Chen $Q$, Jiang $Z$, Xu G X, Zhang X C. Near-field terahertz imaging with a dynamic aperture. Opt Lett 25, 1122-1124 (2000)
48. Busch S, Scherger B, Scheller M, Koch M. Optically controlled terahertz beam steering and imaging. Opt Lett 37, 1391-1393 (2012).

49. Shrekenhamer D, Watts $C M$, Padilla W J. Terahertz single pixel imaging with an optically controlled dynamic spatial light modulator. Opt Express 21, 12507-12518 (2013).

50. Kannegulla A, Jiang Z, Rahman S M, Shams M I B, Fay P et al. Coded-aperture imaging using photo-induced reconfigurable aperture arrays for mapping terahertz beams. IEEE Trans Terahertz Sci Technol 4, 321-327 (2014).

51. Augustin $\mathrm{S}$, Hieronymus J, Jung $\mathrm{P}$, Hübers $\mathrm{H}$ W. Compressed sensing in a fully non-mechanical $350 \mathrm{GHz}$ imaging setting. $J$ Infrared, Millim Terahertz Waves 36, 496-512 (2015).

52. Shang $Y$ J, Wang $X K$ K, Sun W F, Han P, Ye J S et al. Terahertz image reconstruction based on compressed sensing and inverse Fresnel diffraction. Opt Express 27, 14725-14735 (2019).

53. She R B, Liu W Q, Lu Y F, Zhou Z S, Li G Y. Fourier single-pixel imaging in the terahertz regime. Appl Phys Lett 115, 021101 (2019).

54. Stantchev R I, Yu X, Blu T, Pickwell-MacPherson E. Real-time terahertz imaging with a single-pixel detector. Nat Commun 11, 2535 (2020).

55. Watts C M, Shrekenhamer D, Montoya J, Lipworth G, Hunt J et al. Terahertz compressive imaging with metamaterial spatial light modulators. Nat Photon 8, 605-609 (2014).

56. Kappa J, Sokoluk D, Klingel S, Shemelya C, Oesterschulze E et al. Electrically reconfigurable micromirror array for direct spatial light modulation of terahertz waves over a bandwidth wider than 1 THz. Sci Rep 9, 2597 (2019).

57. Zhao J P, Yiwen E, Williams K, Zhang X C, Boyd R W. Spatial sampling of terahertz fields with sub-wavelength accuracy via probe-beam encoding. Light Sci Appl 8, 55 (2019).

58. Olivieri L, Totero Gongora J S, Pasquazi A, Peccianti M. Time-resolved nonlinear ghost imaging. ACS Photon 5, 3379-3388 (2018).

59. Olivieri L, Totero Gongora J S, Peters L, Cecconi V, Cutrona A et al. Hyperspectral terahertz microscopy via nonlinear ghost Imaging. Optica 7, 186-191 (2020).

60. Saqueb S A N, Sertel K. Phase-sensitive single-pixel Thz imaging using intensity-only measurements. IEEE Trans Terahertz Sci Technol 6, 810-816 (2016).

61. Shen Y C, Gan L, Stringer M, Burnett A, Tych K et al. Terahertz pulsed spectroscopic imaging using optimized binary masks. Appl Phys Lett 95, 231112 (2009).

62. Zanotto L, Piccoli R, Dong J, Caraffini D, Morandotti R, Razzari L. Time-domain terahertz compressive imaging. Opt Express 28, 3795-3802 (2020).

63. Stantchev R I, Sun B Q, Hornett S M, Hobson P A, Gibson G M et al. Noninvasive, near-field terahertz imaging of hidden objects using a single-pixel detector. Sci Adv 2, e1600190 (2016).

64. Stantchev R I, Phillips D B, Hobson P, Hornett S M, Padgett M J et al. Compressed sensing with near-field $\mathrm{THz}$ radiation. Optica 4, 989-992 (2017).

65. Hornett S M, Stantchev R I, Vardaki M Z, Beckerleg C, Hendry E. Subwavelength terahertz imaging of graphene photoconductivity. Nano Lett 16, 7019-7024 (2016).

66. Stantchev R I, Mansfield J C, Edginton R S, Hobson P, Palombo $\mathrm{F}$ et al. Subwavelength hyperspectral $\mathrm{THz}$ studies of articular cartilage. Sci Rep 8, 6924 (2018).

67. Chen S C, Du L H, Meng K, Li J, Zhai Z H et al. Terahertz wave near-field compressive imaging with a spatial resolution of over 


\section{Opto-Electronic Advances https://doi.org/10.29026/oea.2020.200012}

N/100. Opt Lett 44, 21-24 (2019).

68. Mohr T, Herdt A, Elsässer W. 2D tomographic terahertz imaging using a single pixel detector. Opt Express 26, 3353-3367 (2018).

69. Saqueb S A N, Sertel K. Compressive Terahertz Imaging Using a Single-Bit Sensor. IEEE Trans Terahertz Sci Technol 8, 757-764 (2018).

70. Saqueb S A N, Sertel K. Multisensor compressive sensing for high frame-rate imaging system in the $\mathrm{THz}$ Band. IEEE Trans Terahertz Sci Technol 9, 520-523 (2019).

71. Chen S C, Feng Z, Li J, Tan W, Du L H et al. Ghost spintronic THz-emitter-array microscope. Light Sci App/ 9,99 (2020).

72. Seifert T, Jaiswal S, Martens U, Hannegan J, Braun L et al. Efficient metallic spintronic emitters of ultrabroadband terahertz radiation. Nat Photon 10, 483-488 (2016).

73. Teo S M, Ofori-Okai B K, Werley C A, Nelson K A. Invited Article: Single-shot $\mathrm{THz}$ detection techniques optimized for multidimensional THz spectroscopy. Rev Sci Instrum 86, 051301 (2015).

\section{Acknowledgements}

We are grateful for financial support from the Natural Sciences and Engineering Research Council of Canada (NSERC) (Strategic Grant: Terahertz hyper-imaging systems for advanced manufacturing). We also would like to thank our industrial partners TeTechS, Lumenera, and Integrity Testing Laboratory (ITL) for useful discussions. Note that R.M. is affiliated to (2) as adjoint faculty.

\section{Author contributions}

LZ carried out the literature searches and prepared the first draft of the paper. All authors commented on the manuscript.

\section{Competing interests}

The authors declare no competing financial interests. 